\title{
The role of TolA, TolB, and TolR in cell morphology, OMVs production, and virulence of Salmonella Choleraesuis
}

\author{
Quan $\mathrm{Li}^{1,2}$, Zheng $\mathrm{Li}^{1,2}$, Xia Fei ${ }^{1,2}$, Yichen Tian ${ }^{1,2}$, Guodong Zhou ${ }^{1,2}$, Yuhan $\mathrm{Hu}^{1,2}$, Shifeng Wang ${ }^{3}$ and \\ Huoying Shi ${ }^{1,2,4^{*}}$
}

\begin{abstract}
The Tol-Pal system of Gram-negative bacteria is necessary for maintaining outer membrane integrity. It is a multiprotein complex of five envelope proteins, TolQ, ToIR, TolA, TolB, and Pal. These proteins were first investigated in E. coli, and subsequently been identified in many other bacterial genera. However, the function of the Tol-Pal system in Salmonella Choleraesuis pathogenesis is still unclear. Here, we reported the role of three of these proteins in the phenotype and biology of $S$. Choleraesuis. We found that mutations in tolA, tolB, and tolR caused severe damage to the cell wall, which was supported by observing the microstructure of spherical forms, long chains, flagella defects, and membrane blebbing. We confirmed that all the mutants significantly decreased S. Choleraesuis survival when exposed to sodium deoxycholate and exhibited a high sensitivity to vancomycin, which may be explained by the disruption of envelope integrity. In addition, to $A$, to $B$, and to $R$ mutants displayed attenuated virulence in a mouse infection model. This could be interpreted as a series of defective phenotypes in the mutants, such as severe defects in envelope integrity, growth, and motility. Further investigation showed that all the genes participate in outer membrane vesicles (OMVs) biogenesis. Interestingly, immunization with OMVs from $\triangle$ tolB efficiently enhanced murine viability in contrast to OMVs from the wild-type $S$. Choleraesuis, suggesting its potential use in vaccination strategies. Collectively, this study provides an insight into the biological role of the $S$. Choleraesuis Tol-Pal system.
\end{abstract}

Keywords: Tol-Pal system, tolA, tolB, tolR, Salmonella Choleraesuis, Outer membrane vesicles, Virulence

\section{Introduction}

Salmonella enterica serovar Choleraesuis (S. Choleraesuis), a Gram-negative bacterium, is an important swine pathogen that cause a series of severe diseases, including meningitis, hepatitis, pneumonia, and other systemic diseases (Reed et al. 1986). Moreover, it's a major zoonotic agent that could be occasionally isolated from humans and triggers huge economic damage in the porcine sector across the globe (Allison et al. 1969; Bangtrakulnonth et al. 2004; Gray et al. 1995). Thus far, the pathogenesis

\footnotetext{
${ }^{*}$ Correspondence: hyshi@yzu.edu.cn

${ }^{1}$ College of Veterinary Medicine, Yangzhou University, Yangzhou 225009, Jiangsu, People's Republic of China

Full list of author information is available at the end of the article
}

of $S$. Choleraesuis infections is still not fully understood (Chiu et al. 2004). Hence, it's imperative to elucidate the pathogenic mechanism of $S$. Choleraesuis.

The Tol-Pal system of Gram-negative bacteria is a multiprotein composite traversing the inner membrane, periplasm, and outer membrane (OM) (Hirakawa et al. 2020). It comprises five envelope proteins, corresponding to TolQ, TolR, TolA, TolB, and Pal (Henry et al. 2004). Three inner membrane proteins TolQ, TolA, and TolR exhibit interaction with each other through their trans-membraneous domains (Derouiche et al. 1995). $\mathrm{Pal}$ is an $\mathrm{OM}$ anchored protein interacting with the periplasmic protein TolB (Ray et al. 2000). The system plays numerous biologic functions in Gram-negative bacteria, including cell morphology, sensitivity to bile salts, and
Springer Open
C The Author(s) 2022. Open Access This article is licensed under a Creative Commons Attribution 4.0 International License, which permits use, sharing, adaptation, distribution and reproduction in any medium or format, as long as you give appropriate credit to the original author(s) and the source, provide a link to the Creative Commons licence, and indicate if changes were made. The images or other third party material in this article are included in the article's Creative Commons licence, unless indicated otherwise in a credit line to the material. If material is not included in the article's Creative Commons licence and your intended use is not permitted by statutory regulation or exceeds the permitted use, you will need to obtain permission directly from the copyright holder. To view a copy of this licence, visit http://creativecommons.org/licenses/by/4.0/. 
bacterial virulence (Dubuisson et al. 2005; Lahiri et al. 2011; Paterson et al. 2009). Furthermore, it has also been displayed that inactivation of the tol-pal genes negatively impacts the outer membrane integrity, resulting in increased formation of outer membrane vesicles (OMVs). OMVs primarily comprise phosphatides, periplasm and OM proteins in Gram-negative microbes, and the TolPal system proteins are the essential components of the OMVs. The Tol-Pal system was 1st characterized in $E$. coli (Webster 1991), and subsequently been reported in many other bacterial genera, including Pseudomonas aeruginosa (Dennis et al. 1996), Vibrio cholerae (Heilpern and Waldor 2000), Pseudomonas putida (Llamas et al. 2000), Salmonella Typhimurium (Prouty et al. 2002), Erwinia chrysanthemi (Dubuisson et al. 2005), and Salmonella Typhi (Lahiri et al. 2011). Although some progress has been made in the research of other Salmonella enterica serovars, the function of the Tol-Pal system in $S$. Choleraesuis has not been documented.

Of particular note, data obtained from $S$. Typhimurium or $S$. Typhi (less from other bacteria) may not be inferred directly to $S$. Choleraesuis without experimental results (Nevermann et al. 2019; Urrutia et al. 2014). Compared with $S$. Typhimurium and S. Typhi, S. Choleraesuis behaves obvious differences in terms of disease progression and host range. They are differences in pathogenic mechanisms, probably due to the different molecular functions of some proteins. Lahiri et al. have demonstrated that there is a considerable difference in the sequence of tolA between $S$. Typhi and $S$. Typhimurium (Lahiri et al. 2011). Deletion of tolA of the two serovars exhibits entirely different phenotypes, including membrane organization, detergent resistance, and cell morphology. Nevermann et al. showed that the tolR mutation of $S$. Typhimurium and $S$. Typhi also presents fully differently regarding sensitivity to vancomycin, motility, and OMVs production (Nevermann et al. 2019). These results indicated that an experimental approach has to be implemented to better elucidate the function of Tol-Pal system of $S$. Choleraesuis.

In this study, and with the aim to explore the roles of tolA, tolB, and tolR genes of the Tol-Pal system in $S$. Choleraesuis, we constructed tolA, tolB, and tolR mutants via homologous recombination. Identifying these genes will help us better comprehend the additional roles of the Tol-Pal system that are difficult to observe in other bacterial genera. We found that all these genes are involved in cell morphology, membrane integrity, cell growth, motility, virulence, and OMVs biogenesis. In addition, we also described the immune responses and protective efficacy of $S$. Choleraesuis OMVs in a mouse model. In general, this study expanded our understanding of the biological role of the $S$. Choleraesuis Tol-Pal system.

\section{Materials and methods}

Plasmids, strains, and growth conditions

Plasmid pRE112 and E. coli strain X7213 were kindly offered by Dr. Roy Curtiss III. S. Choleraesuis strain C78-3 (CVCC79103) were bought from China Institute of Veterinary Drugs Control. E. coli X7213 and S. Choleraesuis strains were grown on LB agar plates or in LB broth (OXOID). When required, $25 \mu \mathrm{g} / \mathrm{ml}$ chloromycetin $(\mathrm{Cm})$ or $50 \mu \mathrm{g} / \mathrm{ml}$ diaminopimelic acid was supplemented into the LB media. Plasmids and strains used in this study are presented in Table 1.

\section{Ethics statement}

Female BALB/c mice (6-week-old) were bought from the Comparative Medicine Center of Yangzhou University. The entire murine studies were completed at Yangzhou University and approved by the Administrative Committee for Laboratory Animals of Jiangsu Province

Table 1 Characteristics of the bacterial strains and plasmids used in this study

\begin{tabular}{|c|c|c|}
\hline Strains or plasmids & General characteristics $^{a}$ & Sources or references \\
\hline \multicolumn{3}{|l|}{ Bacterial strains } \\
\hline C78-3 & Wild type, virulent, CVCC79103 & Ji et al. (2015) \\
\hline$\Delta t o / A$ & Isogenic tolA mutant of strain C78-3 & This study \\
\hline$\Delta t o l B$ & Isogenic tolB mutant of strain $C 78-3$ & This study \\
\hline$\Delta t o / R$ & Isogenic tolR mutant of strain C78-3 & This study \\
\hline$x 7213$ & thi-1 thr-1 leuB6 fhuA21 lacY1 gInV44 asdA4 recA1 RP4 2-Tc::Mu pir & Roland et al. (1999) \\
\hline \multicolumn{3}{|l|}{ Plasmids } \\
\hline pRE112 & sacB mobRP4 R6 K oriV oriT; suicide vector; $\mathrm{Cm}^{r}$ & Edwards et al. (1998) \\
\hline pRE112-tolA & Suicide vector for $\triangle$ tolA; $P R E 112$ derivative; $\mathrm{Cm}^{r}$ & This study \\
\hline pRE112-tolB & Suicide vector for $\triangle t o / B$; pRE112 derivative; $\mathrm{Cm}^{r}$ & This study \\
\hline pRE112-to/R & Suicide vector for $\Delta$ tolR; pRE112 derivative; $\mathrm{Cm}^{r}$ & This study \\
\hline
\end{tabular}

${ }^{\mathrm{a}} \mathrm{Cm}^{\mathrm{r}}$ : chloramphenicol resistance 
[permission number SCXK (SU) 2017-0007]. The process complied with the protocols of Jiangsu Laboratory Animal Welfare and Ethical guidelines, and all endeavors were performed for the purpose of minimizing the pain of the mice.

\section{Construction of the tolA, tolB, and tolR mutants}

Three mutations $\triangle$ tolA, $\triangle t o l B$, and $\Delta t o l R$ were applied for $S$. Choleraesuis strain C78-3 using correspondent suicide vectors. The primers used for PCR amplification of DNA fragments corresponding to the upstream and downstream flanking regions of the tolA, tolB, and tolR genes are listed in Table 2. In brief, the upstream flanking regions (L) and downstream flanking regions (R) of the target genes were fused as complete fragments (LR) via overlapping PCR, and then cloned into pRE112 via the SacI and KpnI restriction sites. The mutations were constructed in C78-3 by conjugating with $\mathrm{X} 7213$ carrying suicide plasmids as previously reported (Curtiss et al. 2009; Roland et al. 1999). PCR confirmation of the deletions using two primer sets of flanking regions (A/D) and internal regions $(\mathrm{E} / \mathrm{F})$, and sequencing by Tsingke Biotechnology Co., Ltd. (Beijing, China).

\section{Transmission electron microscopy (TEM)}

TEM analysis was completed to investigate the role of tolA, tolB, and tolR on the morphology of $S$. Choleraesuis as previously described with minor modification
(Elhenawy et al. 2016). In brief, bacterial strains were cultivated in LB liquid medium with shaking at $37{ }^{\circ} \mathrm{C}$ and collected at the mid-exponential phase $\left(\mathrm{OD}_{600}=0.9\right)$. Afterwards, the cells were allowed to absorb onto carbon-coated copper grids and negatively stained with $1 \%$ uranyl acetate. The samples were allowed to air dry and examined with a Tecnai T12 transmission electron microscope.

\section{Analysis of resistance to sodium deoxycholate and vancomycin}

Assays for resistance to deoxycholic acid were performed as previously described (Nevermann et al. 2019). Briefly, bacterial strains were grown in LB to an $\mathrm{OD}_{600}$ of 0.9 and harvested via centrifugating. Then, the cells were cleaned two times with PBS and subjected to resuspension in $0.5 \%$ sodium deoxycholate or PBS at $37{ }^{\circ} \mathrm{C}$ for $2 \mathrm{~h}$. Microbial survival was counted following plating serial dilutions onto LB agar. The survival rate was computed as (CFU in deoxycholic acid/CFU in $\mathrm{PBS}) \times 100 \%$. The antibiotic sensitivity assay of vancomycin was performed as previously described (Li et al. 2019), using Kirby-Bauer disc diffusion technique. The vancomycin disks contained $30 \mu \mathrm{g}$ of the antibiotic. Every experiment was finished in 3 independently performed biology duplicates.

Table 2 Primers used for PCR amplification and detection

\begin{tabular}{|c|c|c|c|c|}
\hline Primers & Sequences $\left(5^{\prime}-3^{\prime}\right)^{a}$ & Function & Length (bp) & $\begin{array}{l}\text { Restriction } \\
\text { enzyme }\end{array}$ \\
\hline tolA-A & CGCAGAGCTCATTATTGAGGTTTCCGGAGTA & Upstream flanking regions of tolA & 301 & Sacl \\
\hline tolA-B & TCTCGGTTCCCAAAAAACTGT & & & \\
\hline tolA-C & ACAGTTTTTTGGGAACCGAGAATACTTTTCTTTATGGAAGTT & Downstream flanking regions of tolA & 322 & \\
\hline tolA-D & CGGGGTACCTACCGCTATTGCGTAAATCTG & & & Kpnl \\
\hline tolA-E & AGGAGCGGTTGAAACAACTTG & Internal regions of tolA & 562 & \\
\hline tolA-F & CTGAGATCGCCAAGCAGATCG & & & \\
\hline tolB-A & CGCAGAGCTCAATGTGTCTTGCATATTAGCCTG & Upstream flanking regions of tolB & 305 & Sacl \\
\hline tolB-B & CATATCTCCCATACCTGGGCCTG & & & \\
\hline tolB-C & CAGGCCCAGGTATGGGAGATATGTAATAATTAATTGATTACTAA & Downstream flanking regions of tolB & 269 & \\
\hline tolB-D & CGGGGTACCACTTGTCGAGATCGAAGTAAA & & & Kpn I \\
\hline tolB-E & TGCGTTATGCAGGTCATACCG & Internal regions of tolB & 415 & \\
\hline tolB-F & TGACCGGAGGCGAGATCCATA & & & \\
\hline tolR-A & CGCAGAGCTCCGTTTCTTGGCACGGTAGGCT & Upstream flanking regions of tolR & 314 & Sacl \\
\hline tolR-B & GGCTTACCCCTTGTTGCTTTC & & & \\
\hline tolR-C & GAAAGCAACAAGGGGTAAGCCAGTCTGCGTCCCGTTGGCTTG & Downstream flanking regions of tolR & 320 & \\
\hline tolR-D & CGGGGTACCGTTGCAGCTTTTTACGCTCTT & & & Kpnl \\
\hline tolR-E & AGGTCGTCGCGAACTTAAGTC & Internal regions of tolR & 351 & \\
\hline tolR-F & AGCGCTTTAATTATTTCATCG & & & \\
\hline
\end{tabular}

${ }^{a}$ Bold nucleotides denote enzyme restriction sites 


\section{Bacterial growth curve assays}

The $S$. Choleraesuis C78-3 and its mutants $(\Delta t o l A, \Delta t o l B$, and $\Delta$ tolR) were cultivated in $\mathrm{LB}$ to an $\mathrm{OD}_{600}$ of 0.9 and added to $50 \mathrm{ml} \mathrm{LB}$ broth (1:200). The cultures were cultivated by vigorous shaking (200 rpm) at $37{ }^{\circ} \mathrm{C}$ for $12 \mathrm{~h}$. The $\mathrm{OD}_{600}$ of C78-3 and three mutants were quantified at $60 \mathrm{~min}$ interval via a spectral photometer (Bio-Rad). Meanwhile, bacteria numbers were counted every hour following plating serial dilutions onto LB agar. Every experiment was finished in 3 independently conducted biology duplicates.

\section{Motility assays}

Motility assays were performed according to a previously described method with minor modification (Morgan et al. 2014). In short, bacterial strains were cultured in LB to an $\mathrm{OD}_{600}$ of 0.9 , and then diluted 1:10 in fresh $\mathrm{LB}$ and $1 \mu \mathrm{l}$ was inoculated on semi-solid (0.5\%) LB agar plates containing $0.02 \%$ arabinose. The plates were cultivated for $5 \mathrm{~h}$ at $37{ }^{\circ} \mathrm{C}$ and cell motility was assessed via the diameter of growth halo $(\mathrm{mm})$. Each assay was performed in triplicate and repeated in 3 independent replicates.

\section{Assessment of $\mathrm{LD}_{50}$ via a mouse model}

To investigate the effect of inactivating tolA, tolB, and tolR on the virulence of $S$. Choleraesuis, the $\mathrm{LD}_{50}$ of C78-3 and three mutants was tested by intraperitoneal challenges with a mouse model. Briefly, bacterial strains were cultivated in $\mathrm{LB}$ to an $\mathrm{OD}_{600}$ of 0.9 and washed twice with PBS. Four groups of BALB/c mice $(n=4)$ were subjected to injection with the doses of $3,3 \times 10^{1}$, $3 \times 10^{2}$, and $3 \times 10^{3} \mathrm{CFU} /$ mouse in $100 \mu \mathrm{l}$ PBS of wildtype strain C78-3. Meanwhile, 12 groups of mice $(n=4)$ were subjected to injection with the doses of $5 \times 10^{3}$, $5 \times 10^{4}, 5 \times 10^{5}$, and $5 \times 10^{6} \mathrm{CFU} /$ mouse in $100 \mu \mathrm{l} \mathrm{PBS}$ of $\Delta t o l A, \Delta t o l B$, or $\Delta t o l R$, respectively. The animals with the corresponding infection were supervised daily for 30 days. $\mathrm{LD}_{50}$ was determined by the approach of Reed and Muench (1938).

\section{Purification and quantification of OMVs}

Outer membrane vesicles (OMVs) of $S$. Choleraesuis C78-3 and its mutants were isolated as previously described (Muralinath et al. 2011). In brief, bacterial strains were cultivated in LB liquid medium $(300 \mathrm{ml})$ at $37{ }^{\circ} \mathrm{C}$ overnight $\left(\mathrm{OD}_{600}=2.1\right)$ and harvested by centrifugation $(12,000 \times g, 10 \mathrm{~min})$. Subsequently, the supernatants were treated with filtration by $0.45 \mu \mathrm{m}$ sterile filtering device (Millipore, USA). OMVs were collected from the supernatant by ultracentrifugation $(150,000 \times g$, $3 \mathrm{~h}, 4{ }^{\circ} \mathrm{C}$ ) and washed once with PBS. OMVs were then purified by density gradient centrifugation $(150,000 \times g$,
$12 \mathrm{~h}, 4{ }^{\circ} \mathrm{C}$ ) on a discontinuous gradient from 20 to $45 \%$ of Optiprep (Axis-Shield). OMVs fractions were pooled and ultracentrifuged again. The vesicles were resuspended in $2 \mathrm{ml} \mathrm{PBS}$ and stored at $-80{ }^{\circ} \mathrm{C}$. The obtained OMVs isolation was analyzed by TEM as previously described (Nevermann et al. 2019). The yield of OMVs from $S$. Choleraesuis C78-3 and its mutants was evaluated by the protein concentration in the OMVs. Quantification of the OMVs concentration was quantified via a BCA protein analysis kit. All OMVs samples from the strains were subjected to purification and quantification at least 3 times. Each OMVs sample $(8 \mu \mathrm{l})$ was separated by $12 \%$ SDS-PAGE, and then the protein profiles of OMVs were visualized using Coomassie Brilliant Blue R-250.

\section{Immunization and challenge of mice}

Five groups of $\mathrm{BALB} / \mathrm{c}$ mice (6-week-old, $\mathrm{n}=5$ ) were immunized with $100 \mu \mathrm{l}$ PBS involving $10 \mu \mathrm{g}$ OMVs via the intraperitoneal route. Intraperitoneal immunizations of $100 \mu \mathrm{l}$ PBS was the negative controls. Booster immunizations were administered 3 weeks posterior to the primary immunization. Blood specimens were harvested 5 weeks posterior to the initial immunization via orbital sinus puncture. Serum IgG were evaluated by ELISA as previously described (Li et al. 2017). Two weeks after the booster immunizations, the animals were treated with $3 \times 10^{6}$ CFU (nearly $100 \times \mathrm{LD}_{50}$ ) of the wild-type C78-3 in $20 \mu \mathrm{l}$ PBS via the oral route. The infected mice were supervised every day for 30 days. The protection assays were finished two times, and the results were integrated for analysis.

\section{Statistical analysis}

The numerical results were assayed via GraphPad Prism (GraphPad Prism 5, GraphPad Software, USA). Unpaired two-tailed Student's $t$-test was employed to evaluate statistic significance. Differences were considered as significant at $P<0.05$. All results were obtained from at least 3 independent replicates, and values were expressed as mean \pm SEM.

\section{Results}

Construction and confirmation of the tolA, tolB, and tolR knockout mutants

To probe the roles of Tol-Pal system in $S$. Choleraesuis, three mutants of $t o l A, t o l B$, and tolR were constructed via homologous recombination. A schematic representation of the homologous recombination strategy is shown (Fig. 1a). The generation of the $t o l A$, tolB, and tolR mutants using a mediator based on the suicide vector pRE112. The tolA, $t o l B$, and tolR mutants were verified via integrated PCR analysis using two pairs of primers, as well as sequencing. As presented in Fig. 1b, there were no fragments of $\triangle t o l A$, 


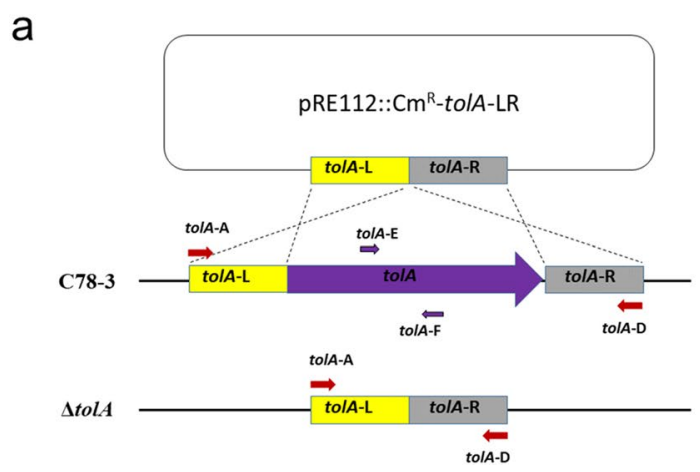

b
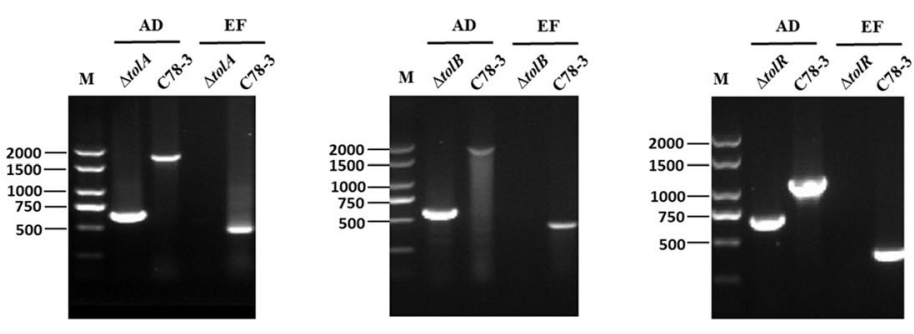

Fig. 1 Construction and confirmation of the to $A$, to $B$, and to $/ R$ knockout mutants. a Schematic diagram for the generation of the to $/ A$, to $/ B$, and tolR mutants. $\mathbf{b}$ The tol $A$, to $B$, and tolR mutants were verified through combined PCR. PCR confirmation of the deletions using two primer sets of flanking regions (A/D) and internal regions (E/F)

$\Delta t o l B$, and $\Delta t o l R$ using inner primers $(\mathrm{E} / \mathrm{F})$, while the flanking primers $(\mathrm{A} / \mathrm{D})$ amplified smaller fragments from the mutants in contrast to those amplified from the parental strain C78-3. These results showed that $\triangle t o l A, \triangle t o l B$, and $\Delta t o l R$ were constructed successfully.

\section{Morphological characterization of S. Choleraesuis C78-3 and three mutants}

Considering that the Tol-Pal system of Gram-negative bacteria toward maintaining outer membrane stability, we speculated that deletion of $t o l A$, tolB, and tolR might influence the phenotypes of $S$. Choleraesuis. Cell morphology of C78-3 and three mutants were examined by light microscopy after Gram staining. In LB broth, the wild-type $S$. Choleraesuis C78-3 grew as single rods (Fig. 2a). Under the same conditions, $\triangle t o l A, \triangle t o l B$, and $\Delta t o l R$ grew in chains (3 to 10 cells) of coccobacilli (Fig. 2a) (red arrow). In order to further confirm the results, we evaluated the cell morphology by TEM. C78-3 was rod-shaped with the average size of $1.6 \times 0.7 \mu \mathrm{m}$ and presented long flagella (blue arrow). In contrast, $\Delta t o l A, \Delta t o l B$, and $\Delta t o l R$ presented an altered morphology with spherical forms and long chains, which is consistent with the above observations (Fig. 2b). Of particular note, the cell morphology among the $\Delta t o l A$, $\Delta t o l B$, and $\Delta t o l R$ strains are very similar. The three mutants formed vesicles at the cell surface (red arrow), but had no flagella. We also found that some mutants were severely damaged in their cell morphology (green arrow). Therefore, $S$. Choleraesuis tolA, tolB, and tolR genes participate in the maintenance of cell morphology.

\section{Characterization of $\Delta$ tolA, $\Delta$ tolB, and $\Delta$ tolR regarding the envelope integrity}

Previous studies found that the tol-pal genes of E. coli are important in maintaining outer membrane integrity (Lazzaroni et al. 1999). This phenomenon was also reported in S. Typhimurium (Paterson et al. 2009) and Erwinia chrysanthemi (Dubuisson et al. 2005). To determine whether deletion of $t o l A, t o l B$, and tolR affect the envelope integrity of $S$. Choleraesuis, assays for resistance to deoxycholic acid were performed. All mutants were more susceptible to $0.5 \%$ sodium deoxycholate in contrast to that of the parental strain (Fig. 3a). To further probe the envelope integrity, the sensitivity of $\triangle t o l A$, $\Delta t o l B$, and $\Delta t o l R$ towards vancomycin were analyzed. In essence, Gram-negative bacteria exhibit resistance to vancomycin due to the limit of diffusible molecules through the microbial envelope (Pimenta et al. 1999). Therefore, the increase in sensitivity to vancomycin can be explained by the increase in permeability. Our results showed that wild-type $S$. Choleraesuis presented full resistance to vancomycin. In contrast, all the mutants revealed complete sensitivity to vancomycin (Fig. 3b). The $t o l A, t o l B$, and tolR mutants exhibited increased susceptibility to sodium deoxycholate and vancomycin, indicating that the envelope integrity might be damaged in these cases.

The roles of tolA, tolB, and tolR in cell growth, motility, and virulence of $S$. Choleraesuis

To investigate the biological roles of tolA, tolB, and tolR in $S$. Choleraesuis, the growth curve, motility and virulence of the wild type C78-3 and mutants were studied. The growth of the $\Delta t o l A, \Delta t o l B$, and $\Delta t o l R$ strains was significantly slower than C78-3 in the exponential phase, while there were no observed growth differences among the three mutants (Fig. 4a). The swimming halo diameter of C78-3, $\Delta$ tolA, $\Delta$ tolB , and $\Delta$ tolR was $12.4 \mathrm{~mm}, 4.7 \mathrm{~mm}$, $4.7 \mathrm{~mm}$, or $5.0 \mathrm{~mm}$, respectively. These results indicated that the motility of $S$. Choleraesuis $\Delta t o l A, \Delta t o l B$, and $\Delta t o l R$ was markedly impaired compared with the wild-type strain (Fig. 4b). We then examined the virulence of C78-3 and 

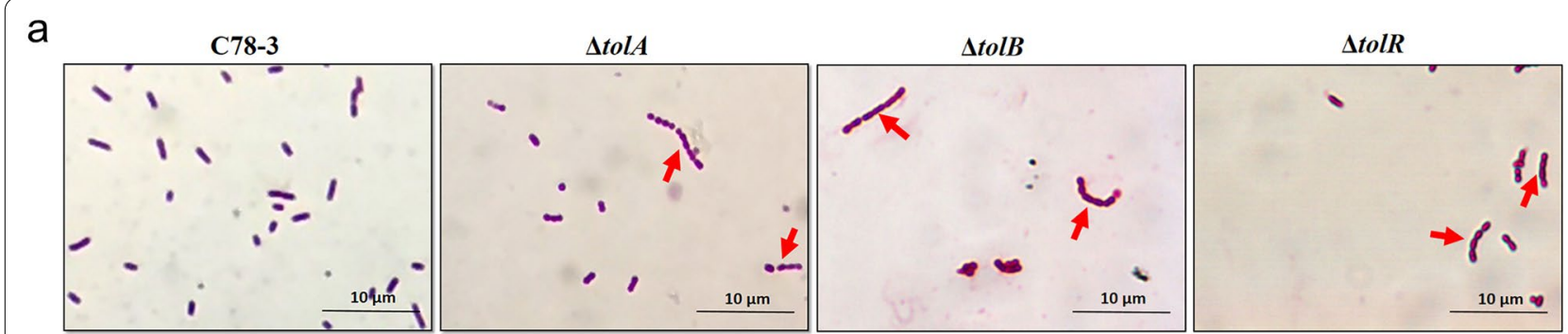

b
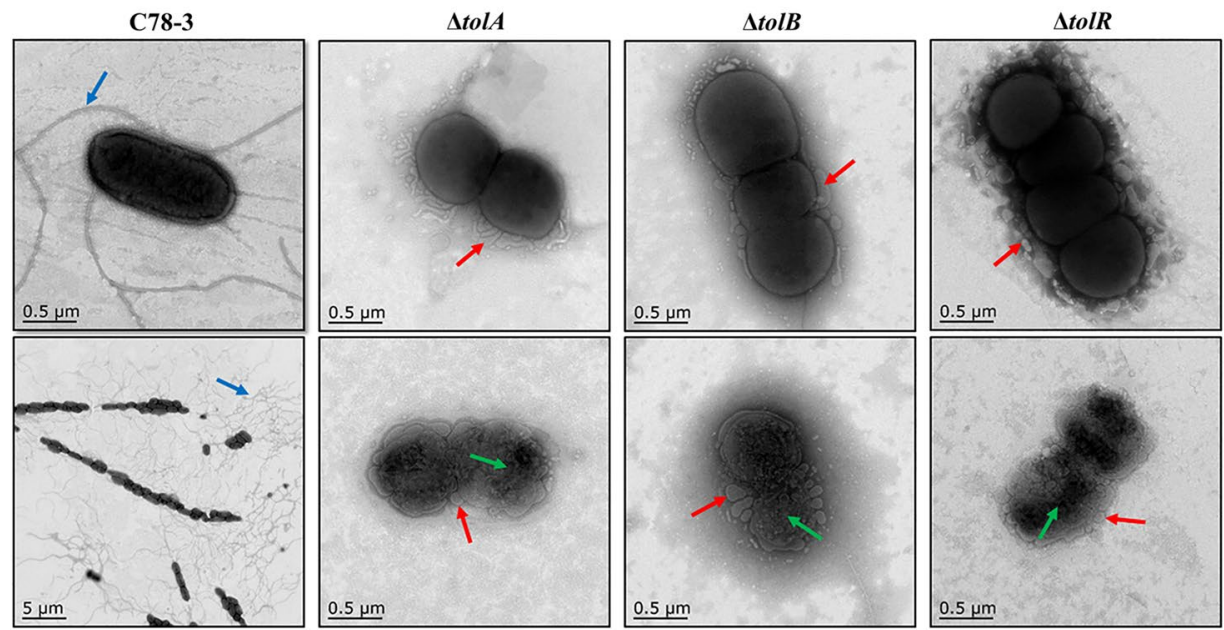

Fig. 2 Morphological characterization of S. Choleraesuis C78-3 and three mutants. a Gram staining of C78-3 and three mutants grown in LB were examined by light microscopy $(\times 1000)$. The $\triangle t o l A, \triangle t o l B$, and $\triangle$ tolR strains grew in chains of coccobacilli (red arrow). $\mathbf{b}$ Morphology characteristics of C78-3 and three mutants were evaluated by TEM. C78-3 was rod-shaped and presented long flagella (blue arrow). The $\Delta$ tolA, $\Delta$ tolB, and $\triangle$ tolR strains showed an altered morphology with long chains and formed vesicles at the cell surface (red arrow), while some mutants were severely damaged in their cell morphology (green arrow)
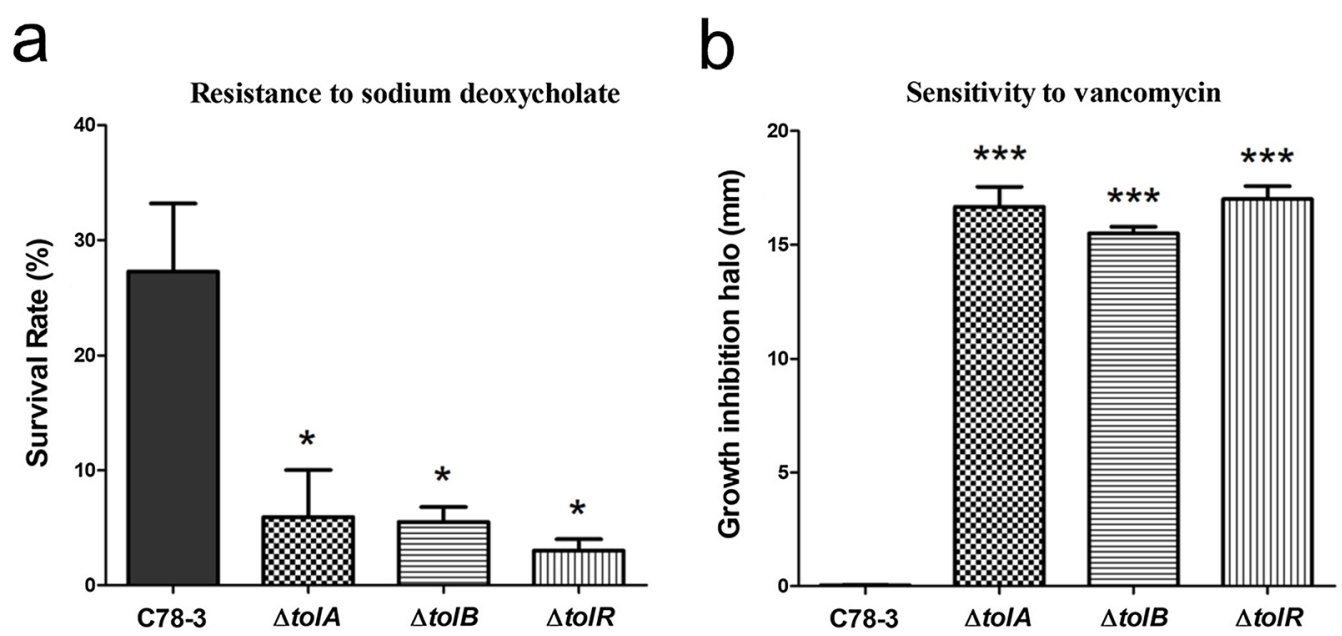

Fig. 3 Resistance to sodium deoxycholate and vancomycin of C78-3 and three mutants. a Survival of C $78-3, \Delta$ tolA, $\Delta$ tolB, and $\triangle$ tolR in $0.5 \%$ sodium deoxycholate. Bacterial strains were cultivated in $\mathrm{LB}$ to an $\mathrm{OD}_{600}$ of 0.9 . Then, the cells were resuspended in $0.5 \%$ sodium deoxycholate or PBS at $37^{\circ} \mathrm{C}$ for $2 \mathrm{~h}$. The survival rate was computed as (CFU in deoxycholic acid/CFU in PBS) $\times 100 \%$. b Resistance to vancomycin of C78-3, $\triangle$ tolA, $\triangle$ tolB, and $\Delta$ tolR strains. The vancomycin disks contained $30 \mu \mathrm{g}$ of the antibiotic. Each assay was completed in 3 independently conducted biology duplicates. ${ }^{*} P<0.05 ;{ }^{* *} P<0.01 ;{ }^{* * *} P<0.001$ 

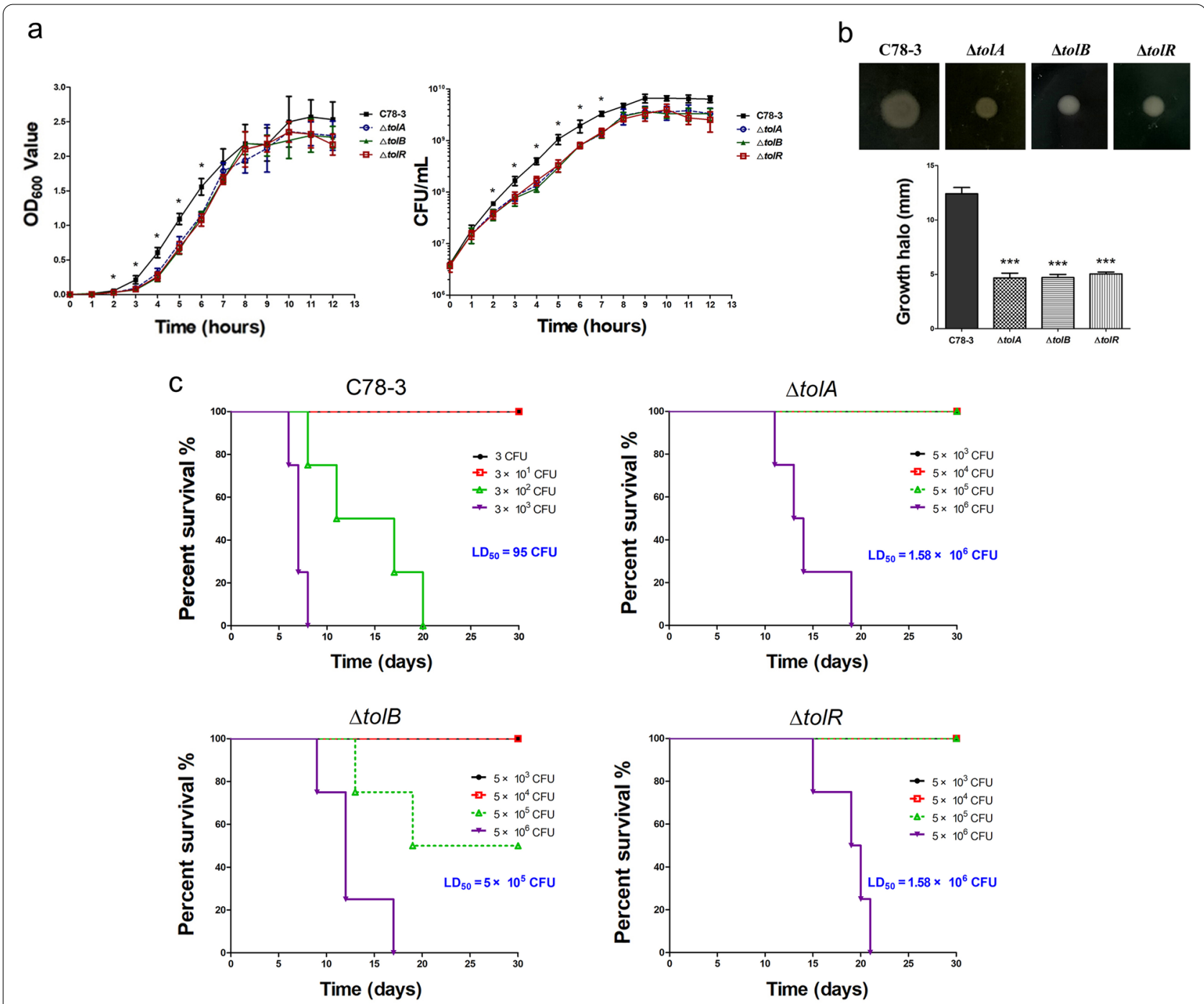

Fig. 4 The roles of tol$A$, tolB, and tolR in cell growth, motility, and virulence of $S$. Choleraesuis. a Growth curves of C78-3, $\Delta t o / A, \Delta$ tolB, and $\Delta t o / R$ using $\mathrm{OD}_{600}$ measurements, and the bacterial suspensions were serially diluted and plated to determine CFU numbers per milliliter. $\mathbf{b}$ Motility was evaluated by measuring the growth halo of $C 78-3, \Delta t o l A, \Delta t o l B$, and $\triangle t o l R$. Each assay was completed in 3 independently conducted biology duplicates. ${ }^{*} P<0.05 ;{ }^{*} P<0.01 ;{ }^{* *} P<0.001$. $\mathbf{c} L D_{50}$ evaluation of $C 78-3, \Delta$ tol $A, \Delta$ tolB, and $\Delta$ tol $R$ with a murine model. The infected mice were supervised every day for 30 days

three mutants in a mouse model through the intraperitoneal route, the $\mathrm{LD}_{50}$ of C78-3, $\triangle$ tolA, $\triangle$ tolB, and $\triangle$ tolR were $95 \mathrm{CFU}, 1.58 \times 10^{6} \mathrm{CFU}, 5 \times 10^{5} \mathrm{CFU}$, or $1.58 \times 10^{6} \mathrm{CFU}$, respectively (Fig. $4 \mathrm{c}$ ). The $\mathrm{LD}_{50}$ value of three $S$. Choleraesuis mutants was significantly higher than that of C78-3. The results suggested that deletion of $t o l A, t o l B$, and tolR displayed an attenuated virulence of $S$. Choleraesuis in a mouse infection model.
The involvement of tolA, tolB, and tolR in OMVs biogenesis of $S$. Choleraesuis

At this point, we speculated that $S$. Choleraesuis tolA, tolB, and tolR genes participate in OMVs biogenesis for the following reasons: (1) all $S$. Choleraesuis tolA, tolB, and tolR mutants showed an impaired envelope integrity compared with the wild-type strain (Fig. 3); (2) vesicles could be clearly observed at the surface of $S$. Choleraesuis 
tolA, tolB, and tolR mutants (Fig. 2b). (3) according to previous reports, tolA, tolB, and tolR genes contribute to the OMVs biogenesis in Salmonella enterica serovars, like tolA and tolB of $S$. Typhimurium (Deatherage et al. 2009), and tolR of $S$. Typhi (Nevermann et al. 2019). To determine whether disruption of tolA, tolB, and tolR

\section{a}

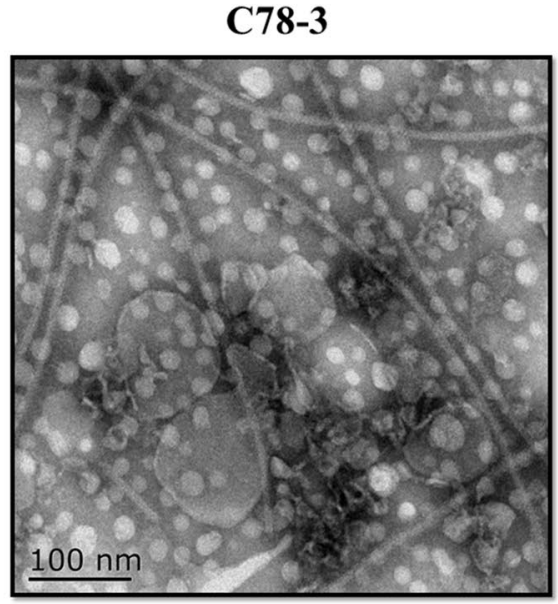

$\Delta t o l B$

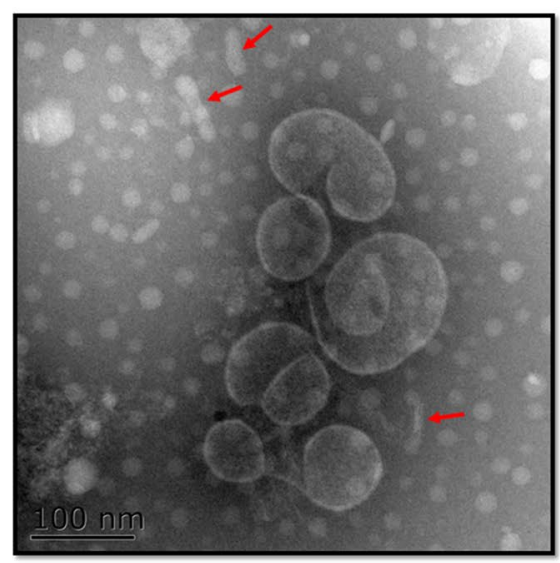

b

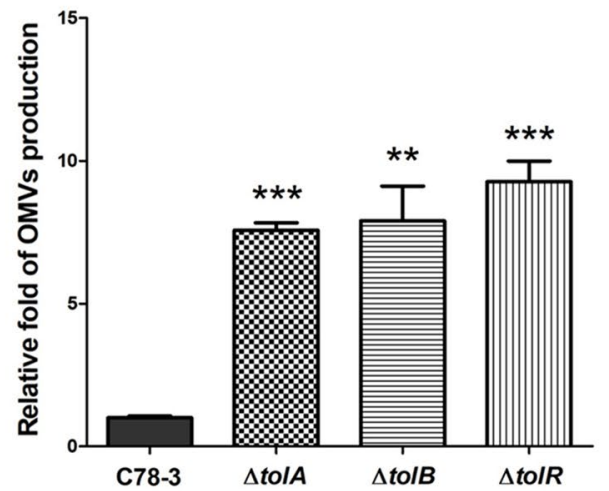

$\Delta t o l A$

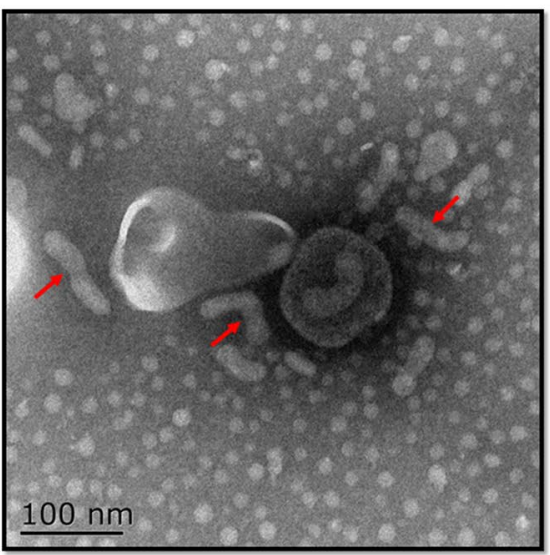

$\Delta t o l R$

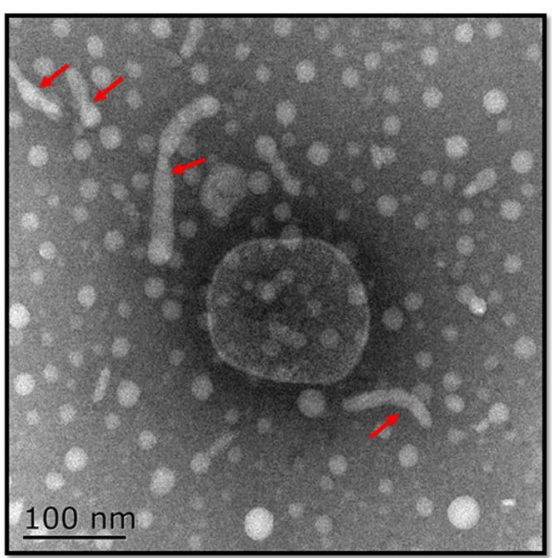

C

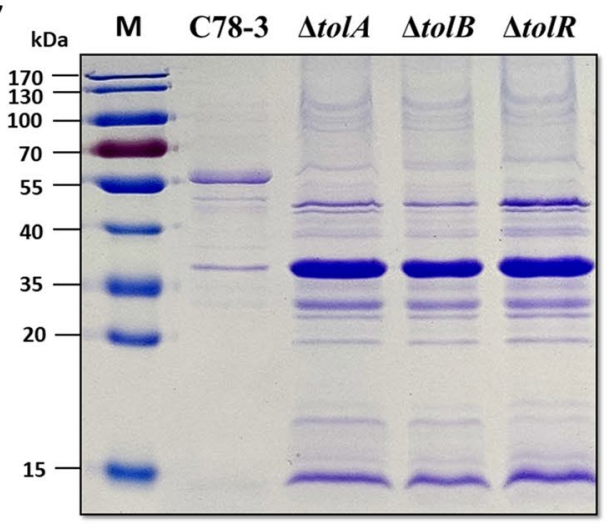

Fig. 5 The involvement of tolA, tolB, and tolR in OMVs biogenesis of $S$. Choleraesuis. a OMVs derived from C78-3, $\Delta$ tol $A, \Delta$ tolB, and $\triangle$ tolR were evaluated by TEM. $\mathbf{b}$ OMVs production of C78-3 and its three mutants. The yield of OMVs was determined by quantitating the protein concentration. The assay was completed in 3 independently conducted biology duplicates. ${ }^{*} P<0.05$; ${ }^{* *} P<0.01$; ${ }^{* *} P<0.001$. c SDS-PAGE profile of OMVs derived from C78-3, $\triangle$ tolA, $\triangle$ tolB, and $\triangle$ tolR strains. Proteins were visualized using Coomassie Brilliant Blue R-250 
influence the OMVs biogenesis of $S$. Choleraesuis, we isolated OMVs from the wild-type and three mutants and further examined by TEM. As shown in Fig. 5a, all $S$. Choleraesuis mutants produced morphologically diverse OMVs regarding their shape (red arrow) and component (with or without flagella) compared with the wild-type strain. The OMVs of wild-type C78-3 has a large number of flagella. In contrast, no obvious flagella were observed in the OMVs preparations of the three mutants, which is consistent with the expressed non motile phenotype observed. In addition, the amount of OMVs in the $\Delta t o l A$, $\Delta t o l B$, and $\Delta$ tolR strains was higher than that in C78-3. To further test the OMVs yield of $S$. Choleraesuis C78-3 and its mutants, we determined the protein concentration of OMVs as an abundance indicator normalizing with $\mathrm{CFU} / \mathrm{ml}$ according to a previous study (Deatherage et al. 2009). Our results showed that the $\Delta t o l A, \Delta t o l B$, and $\Delta t o l R$ strains obviously exhibited more proteins in the OMVs fraction than the wild-type strain (Fig. 5b). Collectively, these data suggested that tolA, tolB, and tolR in $S$. Choleraesuis participate in the OMVs biogenesis.

A previous study demonstrated that OMVs cargo selection is closely related to the OMVs biogenesis (Schwechheimer and Kuehn 2015). Due to tolA, tolB, and tolR gene products are involved in the OMVs biogenesis, we speculated that OMVs cargo selection derived from $S$. Choleraesuis $\Delta$ tolA $\Delta t o l B$, and $\Delta$ tolR strains should be influenced. To examine the differences in OMVs cargo selection, an SDS-PAGE was performed from the OMVs extracts. We observed that $S$. Choleraesuis wild-type OMVs presented few detectable proteins, with a major protein band at $\sim 55 \mathrm{kDa}$ (corresponding to flagellin) (Fig. 5c). The similar pattern of OMVs was also reported in a number of wild-type Salmonella enterica serovars, including $S$. Typhi (Nevermann et al. 2019), S. Typhimurium (Liu et al. 2016a), S. Choleraesuis (Liu et al. 2017b), and $S$. Enteritidis (Liu et al. 2017a). We found that the SDS-PAGE profiles of OMVs derived from $S$. Choleraesuis $\triangle$ tolA $\triangle$ tolB, and $\Delta$ tolR strains (major protein bands at $\sim 45 \mathrm{kDa}, \sim 37 \mathrm{kDa}, \sim 30 \mathrm{kDa}$, and $\sim 15 \mathrm{kDa}$ ) were different from that of $S$. Choleraesuis wild-type OMVs, suggesting the involvement of tolA, tolB, and tolR genes in OMVs cargo selection.

Altogether, these results confirmed that tolA, tolB, and tolR genes participate in the OMVs biogenesis in $S$. Choleraesuis, increasing OMVs production, and affecting OMVs cargo selection.

\section{Evaluation of immune responses and protection against $S$. Choleraesuis}

To investigate the immune responses induced by OMVs, serum IgG was measured by ELISA against outer membrane proteins (OMPs) that were derived from $S$.
Choleraesuis. Five groups of BALB/c mice were subjected to immunization for two times with OMVs or PBS via the intraperitoneal route (Fig. 6a). As shown in Fig. 6b, IgG titers against OMPs from C78-3 and three mutants groups were remarkably higher in contrast to the PBS group. Remarkably, although IgG titers against OMPs from $\triangle t o l A, \Delta t o l B$, and $\Delta t o l R$ groups were slightly higher than the wild-type group, they had no significant difference. Two weeks after the booster immunizations, all animals were challenged with wild-type C78-3 via the oral route. All of the PBS control mice died within 8 days after C78-3 challenge, while immunization with OMVs of C78-3, $\Delta$ tolA, and $\Delta t o l R$ prolonged mice survival to 11 days, 13 days, or 21 days, respectively (Fig. 6c). Specifically, immunization with $\triangle$ tolB OMVs conferred $40 \%$ protection to mice (Fig. 6c), which is significantly higher than that of the wild-type $S$. Choleraesuis OMVs. These data revealed that $\triangle t o l B$ OMVs was able to provide partial protection against the wild-type $S$. Choleraesuis.

\section{Discussion}

The tol-pal genes are not fully characterized in S. Choleraesuis, but they may play important roles in this pathogen considering that the multiple functions of this system in other bacteria. The present study aimed to characterize the function of $t o l A, t o l B$, and tolR genes in S. Choleraesuis. Indeed, tol-pal mutants have been studied extensively in many Gram-negative bacteria (Dennis et al. 1996; Dubuisson et al. 2005; Heilpern and Waldor 2000; Lahiri et al. 2011; Llamas et al. 2000; Prouty et al. 2002; Webster 1991). Mutations in tol-pal genes are impaired in their envelope integrity, affected cell morphology, increased sensitivity to bile salts, promoted OMVs production, reduced the cell growth, motility, and bacterial virulence.

Our analysis confirmed that $S$. Choleraesuis $\Delta t o l A$, $\Delta t o l B$, and $\Delta t o l R$ display an altered cell morphology, which was supported by observing the microstructure of spherical forms, long chains, flagella defects, and membrane blebbing. A phenotype of altered morphology was also reported in tol-pal mutants of $S$. Typhimurium (Masilamani et al. 2018), S. Typhi (Lahiri et al. 2011), and Erwinia chrysanthem (Dubuisson et al. 2005). This phenomenon is mainly due to the disruption of envelope integrity. As previously observed, the Tol-Pal system mediated phosphatidylglycerols trafficking might affect envelope homeostasis that alters cell morphology (Masilamani et al. 2018). Previous studies have demonstrated that the Tol-Pal system of Gram-negative microbes is involved in maintaining $\mathrm{OM}$ integrity (Lazzaroni et al. 1999; Masilamani et al. 2018). According to our results, $\triangle t o l A, \Delta t o l B$, and $\Delta t o l R$ mutants exhibited increased susceptibility to sodium deoxycholate and vancomycin, 

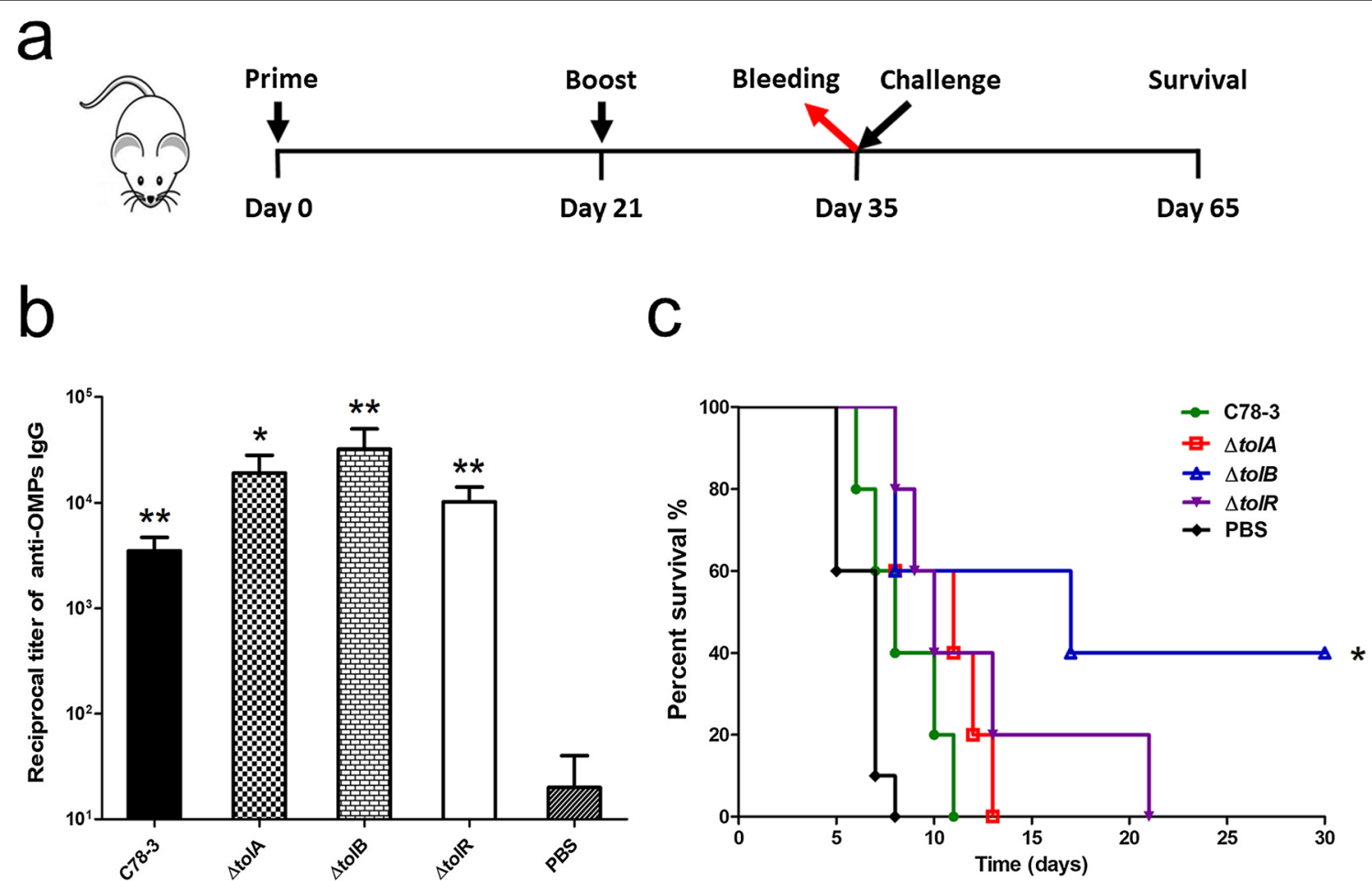

Fig. 6 Evaluation of immune responses and protection against $S$. Choleraesuis. a Scheme of immunization regimen. $\mathbf{b}$ Antibody responses induced by OMVs derived from C78-3, $\triangle$ tolA, $\triangle$ tolB, and $\triangle$ tolR strains. IgG titers against OMPs from C78-3 and three mutants groups were remarkably higher in contrast to the PBS group. c Survival of the mice subjected to immunization with OMVs obtained from C78-3, $\Delta$ tolA, $\Delta$ tolB, and $\triangle$ tolR strains. Intraperitoneal immunizations of PBS served as the negative controls. Two weeks posterior to the reinforce immunizations, all animals were challenged with the wild-type $S$. Choleraesuis. The infected mice were supervised every day for 30 days. ${ }^{*} P<0.05 ;{ }^{* *} P<0.01 ;{ }^{* * *} P<0.001$

revealing that the envelope integrity might be damaged in these cases.

In $S$. Choleraesuis, deletion of tolA, tolB, or tolR is detrimental to the bacteria, since mutants grow very slowly in LB broth. In support of this, the lack of $t o l A$ and $t o l B$ in Erwinia chrysanthemi reduces cell growth (Dubuisson et al. 2005). Our data showed that tolA, tolB, and tolR genes were involved in motility of $S$. Choleraesuis. This phenotype was also observed in tol-pal mutants of $S$. Typhimurium (Nevermann et al. 2019), E. coli (Morgan et al. 2014), and Erwinia chrysanthem (Dubuisson et al. 2005). Our TEM observations provide a valuable explanation for this point. The $t o l A, t o l B$, and $t o l R$ mutations lack flagella, while the wild-type had a large number of flagella around the cell surface (Fig. 2b). By contrast, $S$. Typhi $\Delta$ tolR did not affect the motility (Nevermann et al. 2019), indicating that mutants lacking tolR in different Salmonella enterica serovars are not entirely equivalent.

$S$. Choleraesuis tolA, tolB, and tolR mutants displayed an attenuated virulence in a mouse infection model. Reduced virulence was also reported in tol-pal mutants of other pathogens. In $S$. Typhimurium, the virulence of tolA, $t o l B$, and tolR mutants were significantly attenuated
(Masilamani et al. 2018). In E. coli, the virulence of $\Delta$ tolA was largely attenuated using a Galleria mellonella model (Morgan et al. 2014). In Erwinia chrysanthem, expression of TolA or TolB was necessary for the full virulence in a potato tuber model (Dubuisson et al. 2005). The impaired virulence of $S$. Choleraesuis tolA, tolB, and tolR mutants probably results from the defective phenotypes, such as serious defects in cell morphology, envelope integrity, growth, and motility.

Thus far, the knowledge on the biogenesis of OMVs is still limited and fragmentary in Salmonella serotypes (Deatherage et al. 2009), and significantly lacking in $S$. Choleraesuis. Evidence has revealed that cross-linking between peptidoglycan and bacterial envelope proteins is an important mechanism for OMV biogenesis (Schwechheimer and Kuehn 2015). The Tol-Pal system corresponds to five envelope proteins that participates in OMVs biogenesis by interacting with peptidoglycan. The yield of OMVs increases when the cross-linking decreases. Accordingly, S. Typhimurium tolA and tolB mutants contribute to the production of OMVs (Deatherage et al. 2009). Moreover, the tolR mutation of $S$. Typhi displayed an increased production of OMVs 
(Nevermann et al. 2019). In S. Choleraesuis, we found that $t o l A, t o l B$, and tolR genes participate in the OMVs biogenesis. This study confirmed that TolA, TolB, and TolR are critical cell envelope proteins essential for OMV biogenesis.

Many studies have shown that native OMVs obtained from pathogens were able to confer strong protection against the challenge of pathogenic bacteria, such as $S$. Typhimurium (Liu et al. 2016a, b), S. Enteritidis (Liu et al. 2017a), Shigella flexneri (Camacho et al. 2011), Acinetobacter baumannii (McConnell et al. 2011), Neisseria meningitidis (Serruto et al. 2012). Remarkably, immunization with OMVs isolated from $S$. Choleraesuis wild type via the intraperitoneal route failed to confer protection against $S$. Choleraesuis. This result is likely due to the non-essential immune responses and excessive pro-inflammatory responses (McSorley et al. 2000; Singh et al. 2003; Smith et al. 2003), resulting in the failure of immune protection induced by OMVs. In support of this, similar results has been reported in a previous study (Liu et al. 2017b). Specifically, immunization with OMVs isolated from $\triangle t o l B$ conferred $40 \%$ protection to mice. The result suggested that deletion of $t o l B$ in $S$. Choleraesuis can significantly improve the immunogenicity of OMVs, which is an intriguing discovery for OMVs of $S$. Choleraesuis, and the mechanism needs to be further evaluated.

This study confirmed that deletion of $S$. Choleraesuis tolA, tolB, and tolR genes severely damaged cell morphology, impaired the envelope integrity, inhibited growth and motility ability, and reduced the bacterial virulence. Moreover, tolA, tolB, and tolR genes also participate in the OMVs biogenesis, promoting OMVs production, and affecting OMVs cargo selection. In summary, this study provides an insight into the biological role of the $S$. Choleraesuis Tol-Pal system.

\section{Acknowledgements}

We thank Dr. Roy Curtiss III for kindly providing X7213 and pRE112.

\section{Authors' contributions}

QL was responsible for implementation of the assays, interpreting the data and writing the first draft; $Z L, X F, Y T$, and $G Z$ was responsible for certain assays; SW was involved in the discussion and was responsible for the revision of the first draft; $\mathrm{HS}$ was involved in experiment design was responsible for the interpretation of the data, monitoring the exploration process. All authirs read and approved the final manuscript.

\section{Funding}

This study was supported by the National Natural Science Foundation of China (Grant Numbers 32002301, 32172802, 31672516, 31172300, 30670079), the China Postdoctoral Science Foundation (Grant Number 2019M661953), the Natural Science Foundation of Jiangsu Province (Grant Number BK20190886), and A Project Funded by the Priority Academic Program Development of Jiangsu Higher Education Institutions (PAPD).

Availability of data and materials Not applicable.

\section{Declarations}

Ethics approval and consent to participate

Procedures involving the care and use of animals were approved by the Jiangsu Administrative Committee for Laboratory Animals (permission number SYXK-SU-2007-0005) and complied with the Jiangsu Laboratory Animal Welfare and Ethics guidelines of the Jiangsu Administrative Committee of Laboratory Animals.

\section{Consent for publication}

All authors gave their informed consent prior to their inclusion in the study.

\section{Competing interests}

The authors declare that they have no competing interests.

\section{Author details}

${ }^{1}$ College of Veterinary Medicine, Yangzhou University, Yangzhou 225009, Jiangsu, People's Republic of China. ${ }^{2}$ Jiangsu Co-Innovation Center for the Prevention and Control of Important Animal Infectious Diseases and Zoonoses, Yangzhou 225009, Jiangsu, China. ${ }^{3}$ Department of Infectious Diseases and Immunology, College of Veterinary Medicine, University of Florida, Gainesville, FL 32611-0880, USA. ${ }^{4}$ Joint International Research Laboratory of Agriculture \& Agri-Product Safety (JIRLAAPS), Yangzhou University, Yangzhou 225009, Jiangsu, China.

Received: 16 December 2021 Accepted: 15 January 2022

Published online: 25 January 2022

\section{References}

Allison MJ, Dalton HP, Escobar MR, Martin CJ (1969) Salmonella choleraesuis infections in man: a report of 19 cases and a critical literature review. South Med J 62:593-596

Bangtrakulnonth A, Pornreongwong S, Pulsrikarn C, Sawanpanyalert P, Hendriksen RS, Lo Fo Wong DM, Aarestrup FM (2004) Salmonella serovars from humans and other sources in Thailand, 1993-2002. Emerg Infect Dis 10:131-136

Camacho Al, de Souza J, Sanchez-Gomez S, Pardo-Ros M, Irache JM, Gamazo C (2011) Mucosal immunization with Shigella flexneri outer membrane vesicles induced protection in mice. Vaccine 29:8222-8229

Chiu CH, Su LH, Chu C (2004) Salmonella enterica serotype Choleraesuis: epidemiology, pathogenesis, clinical disease, and treatment. Clin Microbiol Rev 17:311-322

Curtiss R 3rd, Wanda SY, Gunn BM, Zhang X, Tinge SA, Ananthnarayan V, Mo H, Wang S, Kong W (2009) Salmonella enterica serovar typhimurium strains with regulated delayed attenuation in vivo. Infect Immun 77:1071-1082

Deatherage BL, Lara JC, Bergsbaken T, Rassoulian Barrett SL, Lara S, Cookson BT (2009) Biogenesis of bacterial membrane vesicles. Mol Microbiol 72:1395-1407

Dennis JJ, Lafontaine ER, Sokol PA (1996) Identification and characterization of the tolQRA genes of Pseudomonas aeruginosa. J Bacteriol 178:7059-7068

Derouiche R, Benedetti H, Lazzaroni JC, Lazdunski C, Lloubes R (1995) Protein complex within Escherichia coli inner membrane. TolA N-terminal domain interacts with TolQ and TolR proteins. J Biol Chem 270:11078-11084

Dubuisson JF, Vianney A, Hugouvieux-Cotte-Pattat N, Lazzaroni JC (2005) TolPal proteins are critical cell envelope components of Erwinia chrysanthemi affecting cell morphology and virulence. Microbiology (Reading) 151:3337-3347

Edwards RA, Keller LH, Schifferli DM (1998) Improved allelic exchange vectors and their use to analyze 987P fimbria gene expression. Gene 207:149-157

Elhenawy W, Bording-Jorgensen M, Valguarnera E, Haurat MF, Wine E, Feldman MF (2016) LPS remodeling triggers formation of outer membrane vesicles in Salmonella. mBio 7:e00940-16

Gray JT, Fedorka-Cray PJ, Stabel TJ, Ackermann MR (1995) Influence of inoculation route on the carrier state of Salmonella choleraesuis in swine. Vet Microbiol 47:43-59

Heilpern AJ, Waldor MK (2000) CTXphi infection of Vibrio cholerae requires the tolQRA gene products. J Bacteriol 182:1739-1747 
Henry T, Pommier S, Journet L, Bernadac A, Gorvel JP, Lloubes R (2004) Improved methods for producing outer membrane vesicles in Gramnegative bacteria. Res Microbiol 155:437-446

Hirakawa H, Suzue K, Takita A, Awazu C, Kurushima J, Tomita H (2020) Roles of the Tol-Pal system in the Type III secretion system and flagella-mediated virulence in enterohemorrhagic Escherichia coli. Sci Rep 10:15173

Ji Z, Shang J, Li Y, Wang S, Shi H (2015) Live attenuated Salmonella enterica serovar Choleraesuis vaccine vector displaying regulated delayed attenuation and regulated delayed antigen synthesis to confer protection against Streptococcus suis in mice. Vaccine 33:4858-4867

Lahiri A, Ananthalakshmi TK, Nagarajan AG, Ray S, Chakravortty D (2011) TolA mediates the differential detergent resistance pattern between the Salmonella enterica subsp. enterica serovars Typhi and Typhimurium. Microbiology (Reading) 157:1402-1415

Lazzaroni JC, Germon P, Ray MC, Vianney A (1999) The Tol proteins of Escherichia coli and their involvement in the uptake of biomolecules and outer membrane stability. FEMS Microbiol Lett 177:191-197

Li YA, Ji Z, Wang X, Wang S, Shi H (2017) Salmonella enterica serovar Choleraesuis vector delivering SaoA antigen confers protection against Streptococcus suis serotypes 2 and 7 in mice and pigs. Vet Res 48:89

Li Q, Yin J, Li Z, Li Z, Du Y, Guo W, Bellefleur M, Wang S, Shi H (2019) Serotype distribution, antimicrobial susceptibility, antimicrobial resistance genes and virulence genes of Salmonella isolated from a pig slaughterhouse in Yangzhou, China. AMB Express 9:210

Liu Q, Liu Q, Yi J, Liang K, Hu B, Zhang X, Curtiss R 3rd, Kong Q (2016a) Outer membrane vesicles from flagellin-deficient Salmonella enterica serovar Typhimurium induce cross-reactive immunity and provide cross-protection against heterologous Salmonella challenge. Sci Rep 6:34776

Liu Q, Liu Q, Yi J, Liang K, Liu T, Roland KL, Jiang Y, Kong Q (2016b) Outer membrane vesicles derived from Salmonella Typhimurium mutants with truncated LPS induce cross-protective immune responses against infection of Salmonella enterica serovars in the mouse model. Int J Med Microbiol 306:697-706

Liu Q, Yi J, Liang K, Zhang X, Liu Q (2017a) Outer membrane vesicles derived from Salmonella enteritidis protect against the virulent wild-type strain infection in a mouse model. J Microbiol Biotechnol 27:1519-1528

Liu Q, Yi J, Liang K, Zhang X, Liu Q (2017b) Salmonella choleraesuis outer membrane vesicles: proteomics and immunogenicity. J Basic Microbiol 57:852-861

Llamas MA, Ramos JL, Rodriguez-Herva JJ (2000) Mutations in each of the tol genes of Pseudomonas putida reveal that they are critical for maintenance of outer membrane stability. J Bacteriol 182:4764-4772

Masilamani R, Cian MB, Dalebroux ZD (2018) Salmonella Tol-Pal reduces outer membrane glycerophospholipid levels for envelope homeostasis and survival during bacteremia. Infect Immun 86:e00173-18

McConnell MJ, Rumbo C, Bou G, Pachon J (2011) Outer membrane vesicles as an acellular vaccine against Acinetobacter baumannii. Vaccine 29:5705-5710

McSorley SJ, Cookson BT, Jenkins MK (2000) Characterization of CD4+ T cell responses during natural infection with Salmonella typhimurium. J Immunol 164:986-993

Morgan JK, Ortiz JA, Riordan JT (2014) The role for TolA in enterohemorrhagic Escherichia coli pathogenesis and virulence gene transcription. Microb Pathog 77:42-52

Muralinath M, Kuehn MJ, Roland KL, Curtiss R 3rd (2011) Immunization with Salmonella enterica serovar Typhimurium-derived outer membrane vesicles delivering the pneumococcal protein PspA confers protection against challenge with Streptococcus pneumoniae. Infect Immun 79:887-894

Nevermann J, Silva A, Otero C, Oyarzun DP, Barrera B, Gil F, Calderon IL, Fuentes JA (2019) Identification of genes involved in biogenesis of outer membrane vesicles (OMVs) in Salmonella enterica serovar Typhi. Front Microbiol 10:104

Paterson GK, Northen H, Cone DB, Willers C, Peters SE, Maskell DJ (2009) Deletion of tolA in Salmonella Typhimurium generates an attenuated strain with vaccine potential. Microbiology (Reading) 155:220-228

Pimenta AL, Young J, Holland IB, Blight MA (1999) Antibody analysis of the localisation, expression and stability of HlyD, the MFP component of the E. coli haemolysin translocator. Mol Gen Genet MGG 261:122-132
Prouty AM, Van Velkinburgh JC, Gunn JS (2002) Salmonella enterica serovar typhimurium resistance to bile: identification and characterization of the tolQRA cluster. J Bacteriol 184:1270-1276

Ray MC, Germon P, Vianney A, Portalier R, Lazzaroni JC (2000) Identification by genetic suppression of Escherichia coli TolB residues important for TolBPal interaction. J Bacteriol 182:821-824

Reed LJ, Muench H (1938) A simple method of estimating fifty percent endpoints. Am J Hyg 27:493-497

Reed WM, Olander HJ, Thacker HL (1986) Studies on the pathogenesis of Salmonella typhimurium and Salmonella choleraesuis var kunzendorf infection in weanling pigs. Am J Vet Res 47:75-83

Roland K, Curtiss R 3rd, Sizemore D (1999) Construction and evaluation of a delta cya delta crp Salmonella typhimurium strain expressing avian pathogenic Escherichia coli O78 LPS as a vaccine to prevent airsacculitis in chickens. Avian Dis 43:429-441

Schwechheimer C, Kuehn MJ (2015) Outer-membrane vesicles from Gram-negative bacteria: biogenesis and functions. Nat Rev Microbiol 13:605-619

Serruto D, Bottomley MJ, Ram S, Giuliani MM, Rappuoli R (2012) The new multicomponent vaccine against meningococcal serogroup $B, 4 C M e n B$ : immunological, functional and structural characterization of the antigens. Vaccine 30(Suppl 2):B87-97

Singh SP, Williams YU, Miller S, Nikaido H (2003) The C-terminal domain of Salmonella enterica serovar typhimurium OmpA is an immunodominant antigen in mice but appears to be only partially exposed on the bacterial cell surface. Infect Immun 71:3937-3946

Smith KD, Andersen-Nissen E, Hayashi F, Strobe K, Bergman MA, Barrett SL, Cookson BT, Aderem A (2003) Toll-like receptor 5 recognizes a conserved site on flagellin required for protofilament formation and bacterial motility. Nat Immunol 4:1247-1253

Urrutia IM, Fuentes JA, Valenzuela LM, Ortega AP, Hidalgo AA, Mora GC (2014) Salmonella Typhi shdA: pseudogene or allelic variant? Infect Genet Evol 26:146-152

Webster RE (1991) The tol gene products and the import of macromolecules into Escherichia coli. Mol Microbiol 5:1005-1011

\section{Publisher's Note}

Springer Nature remains neutral with regard to jurisdictional claims in published maps and institutional affiliations.

\section{Submit your manuscript to a SpringerOpen ${ }^{\circ}$ journal and benefit from:}

- Convenient online submission

- Rigorous peer review

- Open access: articles freely available online

- High visibility within the field

- Retaining the copyright to your article

Submit your next manuscript at $\mathbf{s p r i n g e r o p e n . c o m ~}$ 